\title{
Widely linear UKF constant modulus algorithm for blind adaptive beamforming
}

\author{
Huaming Qian ${ }^{1}$, Ke Liu ${ }^{1,2, *}$, Long Li ${ }^{3}$, Linchen Qian ${ }^{4}$, and Junda Ma ${ }^{1}$ \\ 1. College of Automation, Harbin Engineering University, Harbin 150001, China; \\ 2. Advanced Signal Processing Lab, College of Engineering, Temple University, Philadelphia, PA 19122, USA; \\ 3. Information Communication Branch, State Grid Heilongjiang Electric Power Company Limited, Harbin 150090, China; \\ 4. School of Computing, Beihang University, Beijing 100191, China
}

\begin{abstract}
Based on a uniform linear array, a new widely linear unscented Kalman filter-based constant modulus algorithm (WLUKF-CMA) for blind adaptive beamforming is proposed. The new algorithm is designed according to the constant modulus criterion and takes full advantage of the noncircular property of the signal of interest (SOI), significantly increasing the output signal-tointerference-plus-noise ratio (SINR), enhancing the convergence speed and decreasing the steady-state misadjustment. Since it requires no known training data, the proposed algorithm saves a large amount of the available spectrum. Theoretical analysis and simulation results are presented to demonstrate its superiority over the conventional linear least mean square-based CMA (LLMS-CMA), the conventional linear recursive least square-based CMA (L-RLS-CMA), WL-LMS-CMA, WL-RLS-CMA and L-UKFCMA.
\end{abstract}

Keywords: widely linear filtering, blind beamforming, noncircular signals, constant modulus algorithm, unscented Kalman filtering.

DOI: $10.21629 /$ JSEE.2017.03.01

\section{Introduction}

Widely linear adaptive beamforming algorithms [1-7] have attracted considerable attention in the last twenty years due to their higher output signal-to-interferenceplus-noise ratio (SINR) and better interference suppression capability than the conventional linear counterparts. In general, it is assumed the received data $x$ to be the second-order circular. In this occasion, the second-order statistics of $\boldsymbol{x}$ can be fully described by the covariance matrix $\boldsymbol{R}=\mathrm{E}\left[\boldsymbol{x} \boldsymbol{x}^{\mathrm{H}}\right]$. However, there are numerous noncircular signals [8], such as binary phase-shift keying (BPSK),

Manuscript received April 10, 2016.

*Corresponding author.

This work was supported by the National Natural Science Foundation of China (61573113), the Harbin Science and Technology Innovation Talents (Excellent Discipline Leader) Research Fund (2014RFXXJ074) and the National Scholarship ([2016]3100). amplitude-shift keying (ASK), unbalanced quaternary phase shift keying (UQPSK) and amplitude modulated (AM). The conventional linear beamformer has been shown to be suboptimal for noncircular signal processing [9]. Therefore, apart from using the covariance matrix $\boldsymbol{R}$, the pseudo-covariance $\widetilde{\boldsymbol{R}}=\mathrm{E}\left[\boldsymbol{x} \boldsymbol{x}^{\mathrm{T}}\right]$ is also considered to characterize the second-order behavior of $\boldsymbol{x}$ for widely linear (WL) beamforming.

Many promising criteria including the minimum variance (MV) [10,11], minimum mean squared error [12,13] (MMSE) and constant modulus (CM) [14] are used to design a WL adaptive beamformer. In this paper, we focus on the scenario where the CM criterion is applied to WL blind adaptive beamforming system because it can form the array response of the signal of interest (SOI) without knowing the training data, and furthermore the $\mathrm{CM}$ criterion-based methods have better performance than the MV criterion-based ones $[15,16]$. Owing to the lowcomplexity and real-time operation, various adaptive filtering algorithms combine the above design criteria to obtain the weight vector of adaptive beamformer. In these existing methods, least mean square (LMS) $[17,18]$ has a low complexity but suffers a slow convergence. Recursive least square (RLS) [19-21] exhibits a fast convergence but needs a high complexity. The conjugate gradient [22,23] (CG) provides a trade-off performance between LMS and RLS. Additional attractive adaptive filtering methods are the Kalman filter-type algorithms [2426]. Especially, since it has superior precision and simplicity of operation, unscented Kalman filter (UKF) has been widely used in some state-estimated scenarios [27]. Afterwards, Bhotto [28] applied UKF to the blind beamforming and demonstrated linear UKF-based constant modulus algorithm (L-UKF-CMA) had performance improvement in the output SINR and convergence speed compared with linear recursive least square-based CMA (L- 
RLS-CMA) and linear least mean square-based CMA (LLMS-CMA).

Recently, exploiting the second-order statistical information of the noncircular signals, Zhang et al. combined RLS with CM criterion to propose WL-RLS-CMA applied to blind equalization, improving equalization performance compared with the traditional L-RLS-CMA algorithm [14]. It is well known that RLS is a special case of Kalman filter and the latter has a better estimated accuracy. Moreover, to the best of our knowledge, there has been no work focusing on Kalman filter applied to adaptive WL beamforming. Therefore, inspired by [14,28], we propose the widely linear unscented Kalman filter-based constant modulus algorithm (WL-UKF-CMA) for WL blind adaptive beamforming and show its performance improvement compared with the existing methods.

In this paper, we make full use of the second-order noncircular property and CM feature of the SOI to propose a new WL blind adaptive beamforming algorithm, i.e., WL-UKF-CMA. Unlike the MMSE-type [29] and linear constraint-type $[30,31]$ methods, the proposed algorithm belongs to a blind beamforming method and does not require a priori information about the reference data or the direction of arrival (DOA) of the SOI, and hence it can save a large amount of the available spectrum. Especially in some scenarios where the users move rapidly or the channel variation is severe, the proposed algorithm has the widespread application value. Theoretical analysis and simulation results show that WL-UKF-CMA is superior to the existing linear and WL blind adaptive beamforming methods in terms of output SINR, convergence speed and mean square error (MSE).

The rest of this paper is organized as follows. The array received model and related problem statement are presented in Section 2. Section 3 details the proposed algorithm and its implementation. In Section 4, performance analyses are described. Section 5 provides extensive numerical results. Finally, we draw the conclusions in Section 6 .

The following notations are used throughout the paper. The superscripts $|\cdot|,(\cdot)^{-1},(\cdot)^{\mathrm{T}},(\cdot)^{*}$ and $(\cdot)^{\mathrm{H}}$ stand for absolute value, matrix inverse, transpose, conjugate and conjugate-transpose, respectively. The expectation is expressed by $\mathrm{E}[\cdot]$. The operation $\mathrm{R}\{\cdot\}$ stands for taking the real part of a variable. $\mathbf{1}_{L \times G}$ and $\mathbf{0}_{L \times G}$ represent the $L \times G$ all-ones and all-zeros vector, respectively. $\boldsymbol{I}_{L}$ is an $L \times L$ dimensional identity matrix.

\section{Array model and problem statement}

\subsection{Array model}

Let us assume $q$ narrowband signals impinge on the uni- form linear array (ULA) equipped with $N$ identical sensors along the DOAs $\theta_{0}, \ldots, \theta_{q-1}$. Then, the observed vector $\boldsymbol{x}(k) \in \mathbf{C}^{N \times 1}$ can be modeled as

$$
\boldsymbol{x}(k)=\sum_{i=0}^{q} \boldsymbol{a}_{i} \boldsymbol{s}_{i}(k)+\boldsymbol{n}(k)
$$

where $\boldsymbol{a}_{i}=\frac{1}{\sqrt{N}}\left[1, \mathrm{e}^{-2 \pi \mathrm{j} \frac{d}{\lambda} \cos \theta_{i}}, \ldots, \mathrm{e}^{-2 \pi \mathrm{j}(N-1) \frac{d}{\lambda} \cos \theta_{i}}\right]^{\mathrm{T}}$ is the normalized steering vector corresponding to the signal $s_{i}, d=\frac{\lambda}{2}$ is the inter element distance of the ULA, $\lambda$ is the wavelength of the signals, $\boldsymbol{n}(k)$ is the additive noise vector which is assumed to be temporally and spatially white Gaussian process with mean 0 and variance $\sigma_{n}^{2}, k$ is the snapshot number. Without loss of generality, in this paper, we assume that $\boldsymbol{s}_{0}$ and $\theta_{0}$ are the SOI and its DOA, respectively, and only the SOI is a constant modulus signal.

\subsection{WL adaptive beamformer}

In order to take full advantage of the second-order noncircular property of the SOI, the augmented observed vector $\overline{\boldsymbol{x}}(k)$ is often used to replace $\boldsymbol{x}(k)$ and can be expressed as

$$
\overline{\boldsymbol{x}}(k)=\left[\boldsymbol{x}^{\mathrm{T}}(k), \boldsymbol{x}^{\mathrm{H}}(k)\right]^{\mathrm{T}} \in \mathbf{C}^{2 N \times 1} .
$$

The corresponding augmented array covariance matrix $\overline{\boldsymbol{R}}$ is made of the covariance matrix $\boldsymbol{R}=\mathrm{E}\left[\boldsymbol{x}(k) \boldsymbol{x}^{\mathrm{H}}(k)\right]$ and the pseudo-covariance matrix $\widetilde{\boldsymbol{R}}=\mathrm{E}\left[\boldsymbol{x}(k) \boldsymbol{x}^{\mathrm{T}}(k)\right]$, and can be expressed as

$$
\overline{\boldsymbol{R}}=\left[\begin{array}{cc}
\boldsymbol{R} & \widetilde{\boldsymbol{R}} \\
\widetilde{\boldsymbol{R}}^{*} & \boldsymbol{R}
\end{array}\right] \in \mathbf{C}^{2 N \times 2 N}
$$

In general, $\boldsymbol{R}$ and $\widetilde{\boldsymbol{R}}$ can be estimated from $L$ snapshots by

$$
\begin{aligned}
& \boldsymbol{R}=\frac{1}{L} \sum_{k=1}^{L} \boldsymbol{x}(k) \boldsymbol{x}^{\mathrm{H}}(k) \\
& \widetilde{\boldsymbol{R}}=\frac{1}{L} \sum_{k=1}^{L} \boldsymbol{x}(k) \boldsymbol{x}^{\mathrm{T}}(k) .
\end{aligned}
$$

As $\boldsymbol{R}$ and $\widetilde{\boldsymbol{R}}$ cannot be obtained directly in practice. The reason that the observed vector $\boldsymbol{x}(k)$ and its covariance matrix $\boldsymbol{R}$ need to be augmented can be explained as follows. The noncircular desired signal can be decomposed [9] as

$$
\boldsymbol{s}_{0}(k)=\widetilde{s}_{0}(k) \mathrm{e}^{\mathrm{j} \varphi_{s}}
$$

where $\widetilde{s}_{0}(k)$ is a real-value symbol, and $\varphi_{s}$ presents the shift phase. According to (6), we know that the observed signal and its conjugated version are correlated. Therefore, the augmented observed vector $\overline{\boldsymbol{x}}(k)$, composed of the observed vector $\boldsymbol{x}(k)$ and its conjugated version $\boldsymbol{x}^{*}(k)$, needs to replace $\boldsymbol{x}(k)$ for WL signal processing. On the 
other hand, since the conjugated observed signal also has useful information of the SOI, which means the pseudocovariance $\widetilde{\boldsymbol{R}}=\mathrm{E}\left[\boldsymbol{x}(k) \boldsymbol{x}^{\mathrm{T}}(k)\right] \neq 0$, the augmented covariance matrix $\overline{\boldsymbol{R}}$ should be used to describe the secondorder noncircular property of SOI instead of $\boldsymbol{R}$.

The output of WL adaptive beamformer $\bar{y}(k)$ can be presented as

$$
\bar{y}(k)=\overline{\boldsymbol{w}}^{\mathrm{H}}(k-1) \overline{\boldsymbol{x}}(k)
$$

where $\overline{\boldsymbol{w}}(k-1)=\left[\boldsymbol{w}^{\mathrm{T}}(k-1), \boldsymbol{w}^{\mathrm{H}}(k-1)\right]^{\mathrm{T}} \in \mathbf{C}^{2 N \times 1}$ is the augmented weight vector of WL adaptive beamformer. The WL adaptive beamformer structure is shown in Fig. 1.

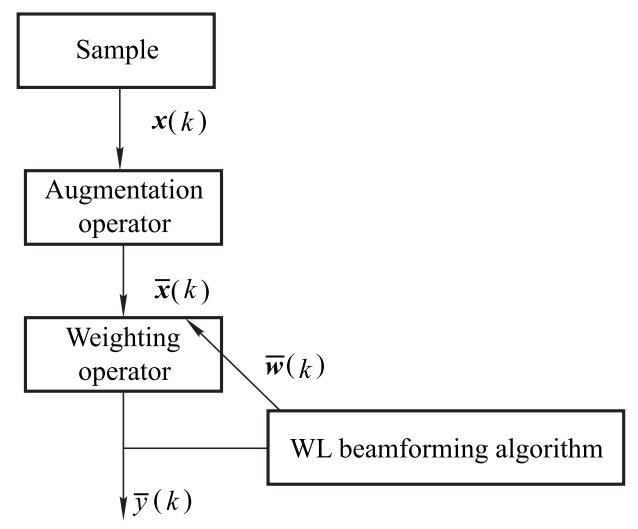

Fig. 1 WL adaptive beamformer structure

\subsection{CMA and problem statement}

CMAs make use of the CM feature of the SOI to suppress the low modulus interference. They obtain the optimal weight vector of the adaptive beamformer by minimizing the mean $p_{3}$ th power error between the array output and a fixed modulus value. The cost function of CMAs $[14,21]$ can be written as

$$
J\left(\overline{\boldsymbol{w}}, p_{1}, p_{2}, p_{3}\right)=\min \mathrm{E}\left[\left(|\bar{y}(k)|^{p_{1}}-p_{2}\right)^{p_{3}}\right]
$$

where $p_{2}$ is the CM value of the SOI, $p_{1}$ and $p_{3}$ are the nonnegative parameters. Our interests focus on how to obtain the weight vector of WL beamformer for blind beamforming in the case $p_{1}=2, p_{2}=1$, and $p_{3}=2$.

In WL-LMS-CMA, by using an instantaneous estimation of the cost function (8), the weight vector is recursively calculated by

$$
\boldsymbol{w}(k+1)=\boldsymbol{w}(k)-\mu\left(|y(k)|^{2}-1\right) y^{*}(k) \boldsymbol{x}(k)
$$

where $\mu$ presents a step size parameter and is usually a small positive value.

In WL-RLS-CMA, the cost function (8) is replaced by an exponentially weighted sum of the square error which is written as

$$
J(\boldsymbol{w})=\sum_{i=1}^{k} \lambda^{k-i}\left(\left|\boldsymbol{w}^{\mathrm{H}}(k) \boldsymbol{x}(i)\right|^{2}-1\right)^{2}
$$

where $\lambda \in(0,1]$ is the forgetting factor. In order to solve (10), when $i$ is close to $k$, an approximation that $\boldsymbol{x}^{\mathrm{H}}(i) \boldsymbol{w}(k) \approx \boldsymbol{x}^{\mathrm{H}}(i) \boldsymbol{w}(i-1)$ is given. Then, under this assumption, we can obtain that

$$
\begin{gathered}
J(\boldsymbol{w})=\sum_{i=1}^{k} \lambda^{k-i}\left[\boldsymbol{w}^{\mathrm{H}}(k) \boldsymbol{x}(i) \boldsymbol{x}^{\mathrm{H}}(i) \boldsymbol{x}(k)-1\right]^{2} \approx \\
\sum_{i=1}^{k} \lambda^{k-i}\left[\boldsymbol{w}^{\mathrm{H}}(k) \boldsymbol{x}(i) \boldsymbol{x}^{\mathrm{H}}(i) \boldsymbol{w}(i-1)-1\right]^{2}= \\
\sum_{i=1}^{k} \lambda^{k-i}\left(\boldsymbol{w}^{\mathrm{H}}(k) \boldsymbol{z}(i)-1\right)^{2}
\end{gathered}
$$

where $\boldsymbol{z}(i)=\boldsymbol{x}(i) \boldsymbol{x}^{\mathrm{H}}(i) \boldsymbol{w}(i-1)$. Now, the weight vector of WL-RLS-CMA can be updated by the conventional RLS. Unlike LMS-type and RLS-type methods, we use UKF to solve the cost function (8) which will be derived in detail in Section 3.

\section{Proposed WL-UKF-CMA algorithm}

\subsection{Deriving of WL-UKF-CMA}

We assume the WL adaptive beamformer is a stationary system. Then, the cost function of CMAs (8) can be converted into the following forms:

$$
\begin{gathered}
\overline{\boldsymbol{w}}(k)=\overline{\boldsymbol{w}}(k-1) \\
1=\left|\overline{\boldsymbol{x}}^{\mathrm{H}}(k) \overline{\boldsymbol{w}}(k-1)\right|^{2} .
\end{gathered}
$$

In order to get a Kalman filter-style state space model, we define that

$$
\begin{gathered}
\boldsymbol{A}(k)=\left(\begin{array}{cc}
\boldsymbol{I}_{2 N \times 2 N} & \mathbf{0}_{2 N \times 1} \\
\overline{\boldsymbol{x}}^{\mathrm{H}}(k) & 0
\end{array}\right) \in \mathbf{C}^{(2 N+1) \times(2 N+1)} \\
\widehat{\boldsymbol{w}}(k-1)=\left(\begin{array}{c}
\overline{\boldsymbol{w}}(k-1) \\
\bar{y}(k-1)
\end{array}\right) \in \mathbf{C}^{(2 N+1) \times 1} \\
\boldsymbol{q}(k-1)=\left(\begin{array}{c}
\mathbf{0}_{2 N \times 1} \\
q(k-1)
\end{array}\right) \in \mathbf{C}^{(2 N+1) \times 1}
\end{gathered}
$$

Then, (12) and (13) can be expressed as

$$
\begin{gathered}
\widehat{\boldsymbol{w}}(k)=\boldsymbol{A}(k) \widehat{\boldsymbol{w}}(k-1)+\boldsymbol{q}(k-1) \\
\widehat{\boldsymbol{y}}(k)=h(\widehat{\boldsymbol{w}}(k-1))
\end{gathered}
$$

where $h\left(\widehat{\boldsymbol{w}}_{i}(k-1)\right)=\left|\widehat{\boldsymbol{w}}_{i}(k-1)\right|^{2}, \widehat{\boldsymbol{w}}_{i}(k-1)$ is the $i$ th row of $\widehat{\boldsymbol{w}}(k-1)$. Obviously, $\widehat{\boldsymbol{y}}_{2 N+1}(k)=1$ where $\widehat{\boldsymbol{y}}_{2 N+1}(k)$ is the $(2 N+1)$ th row of $\widehat{\boldsymbol{y}}(k)$. Therefore, by using (12), (13), (17) and (18), the Kalman filter-style state space model can be constructed as

$$
\widehat{\boldsymbol{w}}(k)=\boldsymbol{A}(k) \widehat{\boldsymbol{w}}(k-1)+\boldsymbol{q}(k-1)
$$




$$
1=\left|\widehat{\boldsymbol{w}}_{2 N+1}(k)\right|^{2}+\boldsymbol{v}(k)
$$

where (19) and (20) are the system equation and observation equation of Kalman filter, respectively. $\boldsymbol{A}(k)$ is the state-transition matrix. $\boldsymbol{q}(k-1)$ and $\boldsymbol{v}(k)$ are the system noise and measure noise, respectively. In this paper, UKF is used to design the WL adaptive beamformer because of its easiness and reliability.

\subsection{Implementation steps of WL-UKF-CMA}

Initialization [32] $\lambda^{-}=\alpha^{2}(n+\kappa-1)-n+2$ is a scaling constant. $\alpha=1$ is a parameter that determines the spread degree of the sigma points and its value is a small constant $(-4 \leqslant \alpha \leqslant 1$ in general). $\beta=2$ is a constant about the distribution of the system state. $\kappa=0$ is a secondary scaling constant. $n=2 N+1$ is the dimension number of the system state. $\gamma_{1}^{(m)}=\frac{\lambda}{\lambda^{-}+n}, \gamma_{i}^{(m)}=\frac{0.5}{\lambda^{-}+n} \mathbf{1}_{(2 n+1) \times 1}$, $\gamma_{i}^{(c)}=\gamma_{i}^{(m)}, \gamma_{1}^{(c)}=\frac{\lambda^{-}}{\lambda^{-}+n}+1-\alpha^{2}+\beta$, the initial augmented weight vector and augmented covariance matrix are set to $\widehat{\boldsymbol{w}}(0)=\left[\mathbf{1}_{2 N \times 1} ; 0\right]$ and $\boldsymbol{P}(0)=\frac{1}{n} \boldsymbol{I}_{n \times n}$, respectively.

Step 1 Calculate sigma sample points of the UKF.

$$
\begin{gathered}
\chi(k-1 \mid k-1)=\widehat{\boldsymbol{w}}(k-1 \mid k-1) \mathbf{1}_{1 \times(2 n+1)}+\sqrt{\left(n+\lambda^{-}\right)} . \\
{\left[\mathbf{0}_{n \times 1}(\boldsymbol{P}(k-1 \mid k-1))^{1 / 2}-(\boldsymbol{P}(k-1 \mid k-1))^{1 / 2}\right] . \quad(21)}
\end{gathered}
$$

Step 2 Augment the received data $\boldsymbol{x}(k)$ and obtain the augmented state-transition matrix.

$$
\boldsymbol{A}(k)=\left(\begin{array}{cc}
\boldsymbol{I}_{(n-1) \times(n-1)} & \mathbf{0}_{(n-1) \times 1} \\
\overline{\boldsymbol{x}}^{\mathrm{H}}(k) & \mathbf{0}
\end{array}\right) .
$$

Step 3 Sigma sample points are propagated by the following equation:

$$
\operatorname{col}_{i}(\chi(k \mid k-1))=\boldsymbol{A}(k) \operatorname{col}_{i}(\chi(k-1 \mid k-1)) .
$$

where $\operatorname{col}_{i}(\chi(k-1 \mid k-1))(i=1, \ldots, 2 n+1)$ is a function standing for the $i$ th column of $\chi(k-1 \mid k-1)$.

Step 4 Calculate the one-step prediction of the system state and state covariance matrix.

$$
\begin{gathered}
\widehat{\boldsymbol{w}}(k \mid k-1)=\sum_{i=1}^{2 n+1} \gamma_{i}^{(m)} \cdot \operatorname{col}_{i}(\boldsymbol{\chi}(k \mid k-1)) \\
\boldsymbol{P}(k \mid k-1)=\sum_{i=1}^{2 n+1} \gamma_{i}^{(c)}\left[\operatorname{col}_{i}(\boldsymbol{\chi}(k \mid k-1))-\widehat{\boldsymbol{w}}(k \mid k-1)\right] . \\
{\left[\operatorname{col}_{i}(\boldsymbol{\chi}(k \mid k-1))-\widehat{\boldsymbol{w}}(k \mid k-1)\right]^{\mathrm{H}} .}
\end{gathered}
$$

Step 5 Calculate the output of the sigma sample points and obtain the one-step prediction of the system output.

$$
\operatorname{col}_{i}\left(\boldsymbol{y}^{-}(k \mid k-1)\right)=h\left(\operatorname{col}_{i}(\chi(k \mid k-1))\right)
$$

$$
\widehat{\boldsymbol{y}}(k \mid k-1)=\sum_{i=1}^{2 n+1} \gamma_{i}^{(m)} \operatorname{col}_{i}\left(\boldsymbol{y}^{-}(k \mid k-1)\right)
$$

where $h(\cdot)$ is a function and its output is the square operator of the each element.

Step 6 Calculate the observation covariance matrix.

$$
\begin{gathered}
P^{y y}(k \mid k-1)=\sum_{i=1}^{2 n+1} \gamma_{i}^{(c)}\left(\operatorname{col}_{n, i}\left(\boldsymbol{y}^{-}(k \mid k-1)\right)-\right. \\
\left.\widehat{\boldsymbol{y}}_{n, 1}(k \mid k-1)\right) \times\left(\operatorname{col}_{n, i}\left(\boldsymbol{y}^{-}(k \mid k-1)\right)-\right. \\
\left.\widehat{\boldsymbol{y}}_{n, 1}(k \mid k-1)\right)^{\mathrm{H}}+R(k)
\end{gathered}
$$

where $\operatorname{col}_{n, i}\left(\boldsymbol{y}^{-}(k \mid k-1)\right)$ stands for an element, which is located at the ( $n$th row, $i$ th column) of the matrix $\boldsymbol{y}^{-}(k \mid k-1)$.

Step 7 Calculate the noise covariance matrix

$$
R(k)=\left(\frac{1}{2 N} \sum_{i=1}^{2 N} \widehat{\boldsymbol{y}}_{i}(k \mid k-1)\right)^{2}
$$

where $\widehat{\boldsymbol{y}}_{n, 1}(k \mid k-1)$ stands for the $n$th element of the vector $\widehat{\boldsymbol{y}}(k \mid k-1)$. The system and observation noises are adaptively calculated by using the method in [28].

Step 8 Calculate the cross-variance matrix between system state and system output.

$$
\begin{array}{r}
\boldsymbol{P}^{w y}(k \mid k-1)=\sum_{i=1}^{2 n+1} \boldsymbol{\gamma}_{i}^{(c)}\left[\operatorname{col}_{i}(\boldsymbol{\chi}(k \mid k-1))-\right. \\
\widehat{\boldsymbol{w}}(k \mid k-1)] \times\left[\operatorname{col}_{n, i}\left(\boldsymbol{y}^{-}(k \mid k-1)\right)-\widehat{\boldsymbol{y}}_{n, 1}(k \mid k-1)\right]^{\mathrm{H}} .
\end{array}
$$

Step 9 Calculate the filter gain of the UKF.

$$
\boldsymbol{K}_{k}=\boldsymbol{P}^{w y}(k \mid k-1) / P^{y y}(k \mid k-1) .
$$

Step 10 Calculate the optimal augmented system state.

$\widehat{\boldsymbol{w}}(k \mid k)=\widehat{\boldsymbol{w}}(k \mid k-1)+\boldsymbol{K}_{k}\left(1-\widehat{\boldsymbol{y}}_{n}(k \mid k-1)\right)^{*}$.

Step 11 Calculate the augmented error variance matrix.

$$
\boldsymbol{P}(k \mid k)=\boldsymbol{P}(k \mid k-1)-\boldsymbol{K}_{k} P^{y y}(k \mid k-1) \boldsymbol{K}_{k}^{\mathrm{H}} .
$$

Step 12 Get the optimal WL adaptive beamformer and go back to Step 1.

$$
\boldsymbol{w}=\widehat{\boldsymbol{w}}_{k \mid k}(1: n-1) .
$$

\section{Performance analyses}

\subsection{Output SINR analysis}

According to $[33,34]$, the minimum of the CMA roughly corresponds to the one of the MMSE. In other words, the $\mathrm{CM}$ criterion is equivalent to the MMSE one under ideal 
conditions. From [35], we know that the weight vector obtained by using the MMSE criterion approaches the optimal beamformer (optimal MVDR beamformer). Therefore, we can evaluate the output SINR improvement of the proposed WL-UKF-CMA compared to the linear one by analyzing the optimal linear and WL MVDR beamformer. The cost function of the linear MVDR beamformer can be expressed as

$$
\begin{aligned}
& \mathrm{E}\left[|y|^{2}\right]=\boldsymbol{w}^{\mathrm{H}} \boldsymbol{R} \boldsymbol{w} \\
& \text { s.t. } \boldsymbol{w}^{\mathrm{H}} \boldsymbol{a}_{0}=1 .
\end{aligned}
$$

The optimal weight vector $\boldsymbol{w}_{\text {opt }}$ can be obtained as

$$
\boldsymbol{w}_{o p t}^{L}=\frac{\boldsymbol{R}^{-1} \boldsymbol{a}_{0}}{\boldsymbol{a}_{0}^{\mathrm{H}} \boldsymbol{R}^{-1} \boldsymbol{a}_{0}}
$$

by using the Lagrange multiplier method. Obviously, the instantaneous SINR at the output of a linear adaptive beamformer can be expressed as

$$
\operatorname{SINR}^{L}=\frac{\mathrm{E}\left[\left|\boldsymbol{w}^{\mathrm{H}} \boldsymbol{s}_{0}\right|^{2}\right]}{\mathrm{E}\left[\left|\boldsymbol{w}^{\mathrm{H}} \boldsymbol{v}\right|^{2}\right]}
$$

where $\boldsymbol{v}$ is the interference plus noise vector. The optimal output SINR of the linear beamformer can be written as

$$
\operatorname{SINR}_{o p t}^{L}=\frac{\boldsymbol{a}_{0}^{\mathrm{H}} \boldsymbol{R}^{-1} \boldsymbol{a}_{0}}{1-\boldsymbol{a}_{0}^{\mathrm{H}} \boldsymbol{R}^{-1} \boldsymbol{a}_{0}}
$$

Similar to the linear case, the WL optimal MVDR beamformer $\overline{\boldsymbol{w}}_{\text {opt }}$ can be obtained by using the following cost function:

$$
\begin{aligned}
& \mathrm{E}\left[|\bar{y}|^{2}\right]=\overline{\boldsymbol{w}}^{\mathrm{H}} \overline{\boldsymbol{R}} \overline{\boldsymbol{w}} \\
& \text { s.t. } \overline{\boldsymbol{w}}^{\mathrm{H}} \overline{\boldsymbol{a}}_{0}=1
\end{aligned}
$$

where $\overline{\boldsymbol{a}}_{0}=\left[\boldsymbol{a}_{0} ; \boldsymbol{a}_{0}^{*}\right]$. The optimum solution $\overline{\boldsymbol{w}}_{\text {opt }}$ is calculated according to the Lagrange multiplier method and is expressed as

$$
\overline{\boldsymbol{w}}_{o p t}^{W L}=\frac{\overline{\boldsymbol{R}}^{-1} \overline{\boldsymbol{a}}_{0}}{\overline{\boldsymbol{a}}_{0}^{\mathrm{H}} \overline{\boldsymbol{R}}^{-1} \overline{\boldsymbol{a}}_{0}}
$$

The corresponding optimal output SINR is

$$
\operatorname{SINR}_{o p t}^{W L}=\frac{\overline{\boldsymbol{a}}_{0}^{\mathrm{H}} \overline{\boldsymbol{R}}^{-1} \overline{\boldsymbol{a}}_{0}}{1-\overline{\boldsymbol{a}}_{0}^{\mathrm{H}} \overline{\boldsymbol{R}}^{-1} \overline{\boldsymbol{a}}_{0}} .
$$

Given the conjugate symmetry property defined by $\overline{\boldsymbol{w}}^{\mathrm{H}} \overline{\boldsymbol{x}}=\overline{\boldsymbol{x}}^{\mathrm{T}} \overline{\boldsymbol{w}}^{*}=2 \mathrm{R}\left\{\boldsymbol{w}^{\mathrm{H}} \boldsymbol{x}\right\}$, the instantaneous WL output SINR can be expressed as

$$
\operatorname{SINR}^{W L}=\frac{\mathrm{E}\left[\mathrm{R}\left\{\left|\overline{\boldsymbol{w}}^{\mathrm{H}} \overline{\boldsymbol{s}}_{0}\right|^{2}\right\}\right]}{\mathrm{E}\left[\mathrm{R}\left\{\left|\overline{\boldsymbol{w}}^{\mathrm{H}} \overline{\boldsymbol{v}}\right|^{2}\right\}\right]}
$$

where $\overline{\boldsymbol{s}}_{0}=\left[s_{0} ; s_{0}^{*}\right], \overline{\boldsymbol{v}}=\left[\boldsymbol{v} ; \boldsymbol{v}^{*}\right]$. Obviously, the linear optimal MVDR beamformer can be seemed as a special case of WL optimal one in which $\overline{\boldsymbol{w}}=\left[\boldsymbol{w}_{o p t}^{\mathrm{T}}, \mathbf{0}_{1 \times N}\right]^{\mathrm{T}}$. From (41), we can obtain that

$$
\widehat{\mathrm{SINR}}^{L}=\frac{\mathrm{E}\left[\left|\mathrm{R}\left\{\boldsymbol{w}_{o p t}^{\mathrm{H}} \boldsymbol{s}_{0}\right\}\right|^{2}\right]}{\mathrm{E}\left[\left|\mathrm{R}\left\{\overline{\boldsymbol{w}}_{o p t}^{\mathrm{H}} \boldsymbol{v}\right\}\right|^{2}\right]} \leqslant \operatorname{SINR}_{o p t}^{W L} .
$$

Since $R\{\cdot\}$ operation only contains the half of the interference plus noise power, we can derive that $\widehat{\mathrm{SINR}}^{L} \approx$ $\mathrm{SINR}_{\text {opt }}^{L}$. In other words, the WL beamformer can obtain at least $3 \mathrm{~dB}$ gain improvement compared with the linear one which will be verified in Section 5 .

\subsection{Complexity analysis}

The computational complexity of the proposed WL-UKFCMA is of order $O\left(n^{3}\right)$, while those of WL-RLS-CMA and WL-LMS-CMA are $O\left((2 N)^{2}\right)$ and $O(2 N)$, respectively. For their linear counterparts, they are $O\left((N+1)^{3}\right)$, $O\left(N^{2}\right)$ and $O(N)$ for L-UKF-CMA, L-RLS-CMA and LLMS-CMA, respectively. Moreover, we find that LMStype methods need much lower computational loads than RLS-type and UKF-type approaches. Furthermore, it is also easy to see that the WL-based methods are nearly twice more computationally expensive than their corresponding linear ones because the augmented operation increases the array aperture, and the proposed algorithm has the highest complexity among the above methods. However, the proposed WL-UKF-CMA has better performance than other methods mentioned above which will be shown in Section 5.

Remark 1 As UKF does not need to calculate the gradient matrix and can obtain a better estimated accuracy compared to other nonlinear filter methods, e.g. EKF, we adopt UKF to estimate the weight vector of the WL beamformer. Furthermore, the potential reason, that UKF can obtain superior performance, can be explained by the fact that UKF can minimize the mean-phase-error at the same time keeping constant modulus feature of SOI. While the other compared methods, such as RLS-style and LMSstyle approaches, do not have such property.

Remark 2 The proposed WL-UKF-CMA has performance improvement in terms of convergence speed and steady-state SINR since the noncircular property of SOI and WL processing techniques are adopted. Moreover, the system and measure noises are estimated adaptively, which is a difficult point for the conventional KF-style approaches. However, the proposed WL-UKF-CMA owns a higher computational complexity than RLS-style and LMS-style approaches. We could still take some reduceddimension and reduced-rank methods [36-38] to reduce the high computational complexity of WL-UKF-CMA. 
However in this paper, we do not consider adding those methods.

\section{Simulations}

In this section, we evaluate the performance of the proposed WL-UKF-CMA in terms of output SINR and MSE. Firstly, the theoretical analysis about output SINR will be verified by comparing WL-UKF-CMA with L-UKFCMA. Then, WL-UKF-CMA is compared with LMS-type and RLS-type solutions, respectively, to present its superiority. Let us consider a ULA which is composed of $N=5$ identical omnidirectional sensors and whose spacing is half-wavelength. We assume that there are three signals to impinge on the ULA from DOAs $\theta_{0}=10^{\circ}, \theta_{1}=25^{\circ}$ and $\theta_{2}=35^{\circ}$, respectively. Moreover, the SOI is chosen as the BPSK-modulated signal. The signal-to-noise ratio (SNR) and interference-to-noise ratio (INR) are $10 \mathrm{~dB}$ and $3 \mathrm{~dB}$, respectively. The noise power is $\sigma_{n}^{2}=0.1$. All the simulation points are obtained by averaging 50 independent runs.

\subsection{WL-UKF-CMA vs L-UKF-CMA}

In this example, we examine the output SINRs and MSEs of WL-UKF-CMA and L-UKF-CMA in different cases, i.e., Case 1 and Case 2, where the interference signals are circular and noncircular, respectively. In order to verify the theoretical analysis, the linear and WL optimal SINR are provided as well. The circular and noncircular signals correspond to QPSK-modulated and BPSK-modulated ones, respectively.

Fig. 2 presents the output SINR performance of WL-UKF-CMA and L-UKF-CMA versus snapshots in Case 1. From Fig. 2, we see that both WL-UKF-CMA and L-UKF-CMA arrive at the steady-state at around the 400th snapshots, which means they nearly have the same convergence speeds. Moreover, it is also observed that the steadystate output SINRs of WL-UKF-CMA and L-UKF-CMA are about $13 \mathrm{~dB}$ and $10 \mathrm{~dB}$, which nearly approach the WL optimal SINR and linear one, respectively. That is to say that WL-UKF-CMA obtains a higher output SINR than LUKF-CMA. Furthermore, we also find that WL optimal SINR has nearly $3 \mathrm{~dB}$ SINR improvement than the linear one, which is in accordance with the theoretical analysis. The empirical results of the output MSEs of the WL-UKFCMA and L-UKF-CMA in Case 1 are shown in Fig. 3. It is observed in Fig. 3 that WL-UKF-CMA and L-UKFCMA converge at around $-14 \mathrm{~dB}$ and $-12 \mathrm{~dB}$, respectively. That is to say WL-UKF-CMA obtains a lower MSE than L-UKF-CMA. Similar to Fig. 2, WL-UKF-CMA has nearly the same convergence speed with L-UKF-CMA in MSE.

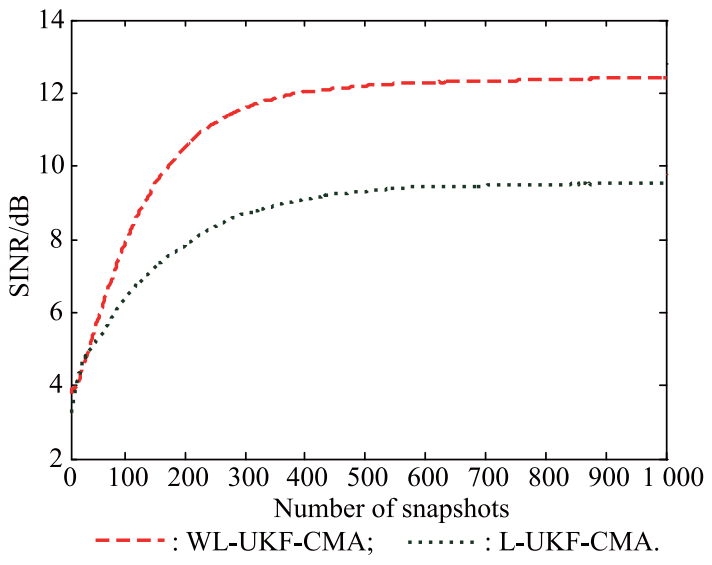

Fig. 2 Output SINRs of WL-UKF-CMA and L-UKF-CMA in Case 1

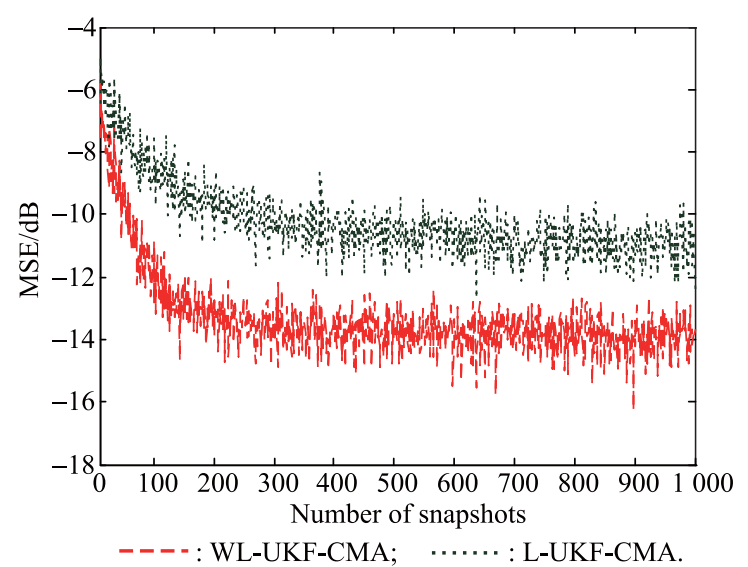

Fig. 3 Output MSEs of WL-UKF-CMA and L-UKF-CMA in Case 1

The learning curves of output SINRs of WL-UKF-CMA and L-UKF-CMA in Case 2 are shown in Fig. 4. We observe from Fig. 4 that WL-UKF-CMA and L-UKF-CMA have a slightly convergence improvement compared with those in Case 2 due to the variation of the interference. In other words, WL-UKF-CMA and L-UKF-CMA converge a little faster than those in Case 1. Similar to Case 2, WLUKF-CMA and L-UKF-CMA approximately converge to the optimal WL SINR and optimal linear SINR, respectively. Fig. 5 shows the output MSEs of WL-UKF-CMA and L-UKF-CMA versus snapshots in Case 2. It is seen in Fig. 5 that WL-UKF-CMA and L-UKF-CMA converge at $-18 \mathrm{~dB}$ and $-12 \mathrm{~dB}$, respectively, which illustrates WLUKF-CMA has a lower MSE than this in Fig. 3 while LUKF-CMA yields the same MSE as this in Case 1. That is due to the fact that WL-UKF-CMA fully exploits the noncircular property of the noncircular signals in Case 2. Moreover, we also see that, in terms of MSE, WL-UKFCMA has a similar convergence speed to L-UKF-CMA. 


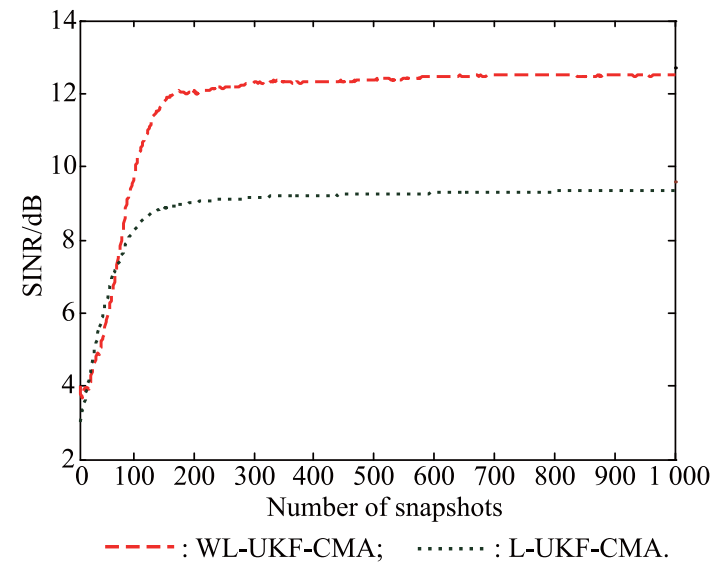

Fig. 4 Output SINRs of WL-UKF-CMA and L-UKF-CMA in Case 2

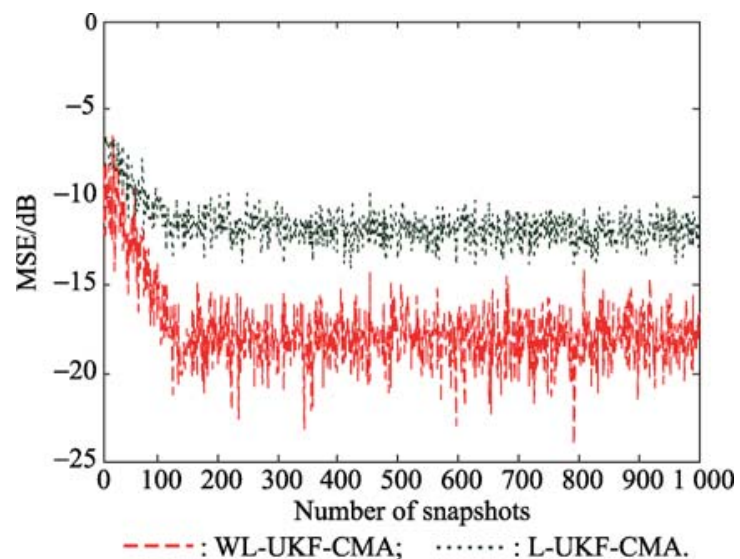

Fig. 5 Output MSEs of WL-UKF-CMA and L-UKF-CMA in Case 2

\subsection{WL-UKF-CMA vs LMS-type methods}

In this example, we show the output SINRs and MSEs of WL-UKF-CMA, WL-LMS-CMA and L-LMS-CMA in Case 1 and Case 2, respectively. To help clarify it, for the WL-based methods, we use "WL-LMS-CMA1" and "WLLMS-CMA2" as the scenarios that the step size $\mu$ is fixed at 0.05 and 0.025 , respectively. In a similar way, "L-LMSCMA1" and "L-LMS-CMA2" correspond to the situations of $\mu=0.05$ and $\mu=0.025$, respectively, for the linearbased schemes.

The output SINRs of WL-UKF-CMA, WL-LMSCMA1, WL-LMS-CMA2, L-LMS-CMA1 and L-LMSCMA2 in Case 1 are displayed in Fig. 6. We see in Fig. 6 that WL-UKF-CMA has nearly the same steadystate output SINR, but a faster convergence speed, compared with WL-LMS-CMA1 and WL-LMS-CMA2. Moreover, we also find that WL-UKF-CMA, WL-LMS-CMA1 and WL-LMS-CMA2 have higher output SINRs than LLMS-CMA1 and L-LMS-CMA2. Furthermore, we observe that, for LMS-type methods, a smaller step size leads to a slower convergence speed but a little higher steady-state output SINR. On the contrary, a larger step size results in a faster convergence speed but a little lower steady-state output SINR. Fig. 7 shows the output MSEs of WL-UKF-CMA, WL-LMS-CMA1, WL-LMS-CMA2, L-LMS-CMA1 and L-LMS-CMA2 versus snapshots in Case 1. We observe in Fig. 7 that WL-UKF-CMA yields a smaller output MSE than its counterparts. What is more, WL-LMS-CMA1 and WL-LMS-CMA2 provide smaller MSEs than L-LMS-CMA1 and L-LMS-CMA2, respectively.

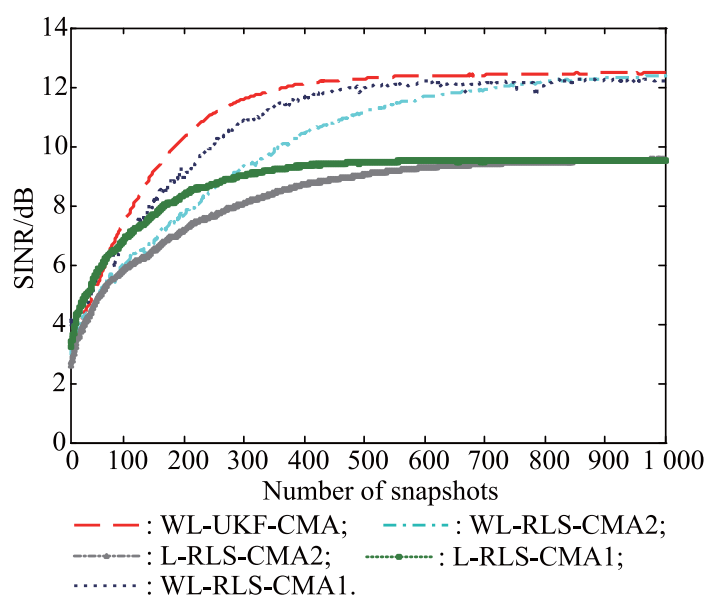

Fig. 6 Output SINRs of WL-UKF-CMA and LMS-type methods in Case 1

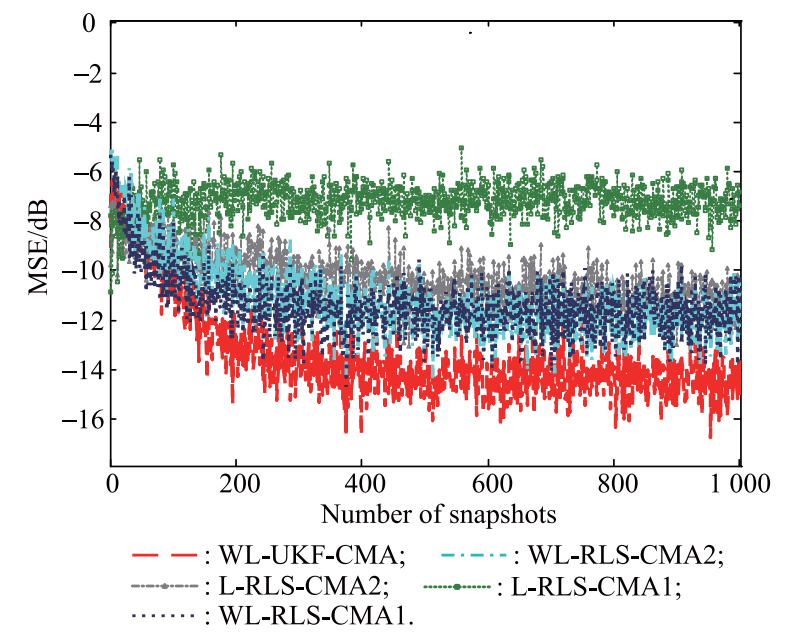

Fig. 7 Output MSEs of WL-UKF-CMA and LMS-type methods in Case 1

Fig. 8 shows the numerical results of output SINRs of WL-UKF-CMA and LMS-type methods (which include WL-LMS-CMA1, WL-LMS-CMA2, L-LMS-CMA1 and L-LMS-CMA2) in Case 2. Similar to Fig. 6, all methods have a slightly convergence improvement due to the variation of the interference signal type, and WLUKF-CMA still has the fastest convergence speed among 
these counterparts. The learning curves of output MSEs of WL-UKF-CMA and LMS-type methods are shown in Fig. 9. From Fig. 9, we see that WL-UKF-CMA nearly has the same steady-state MSE as WL-LMS-CMA1 and WLLMS-CMA2, and L-LMS-CMA1 approximately yields the same steady-state MSE as L-LMS-CMA2. Moreover, the WL-based methods, such as WL-UKF-CMA, WL-LMSCMA1 and WL-LMS-CMA2, have lower MSEs than their corresponding linear solutions, such as L-LMS-CMA1 and L-LMS-CMA2. Furthermore, we also observe that, similar to Fig. 6, WL-LMS-CMA1 and L-LMS-CMA1 have faster convergence speeds and a little higher MSEs than WL-LMS-CMA2 and L-LMS-CMA2, respectively. That is to say that a larger step size leads to a faster convergence speed and a higher MSE, while a smaller step size results in a slower convergence speed and a lower MSE.

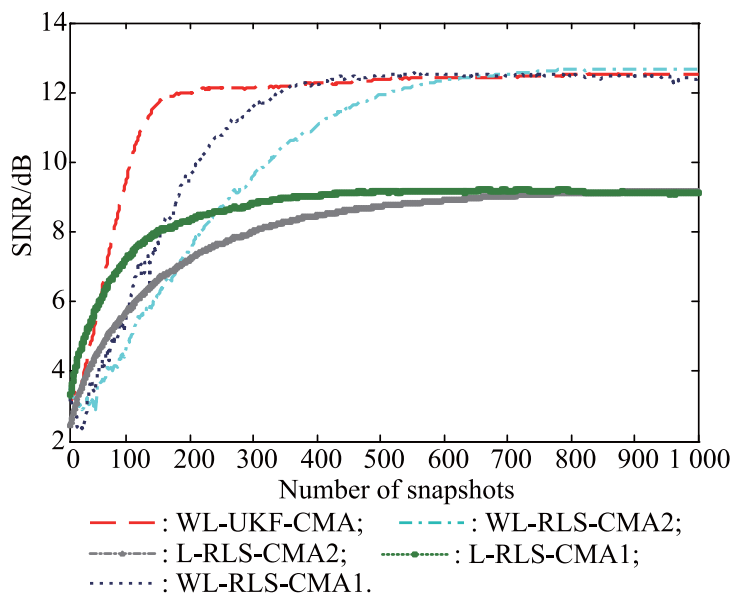

Fig. 8 Output SINRs of WL-UKF-CMA and LMS-type methods in Case 2

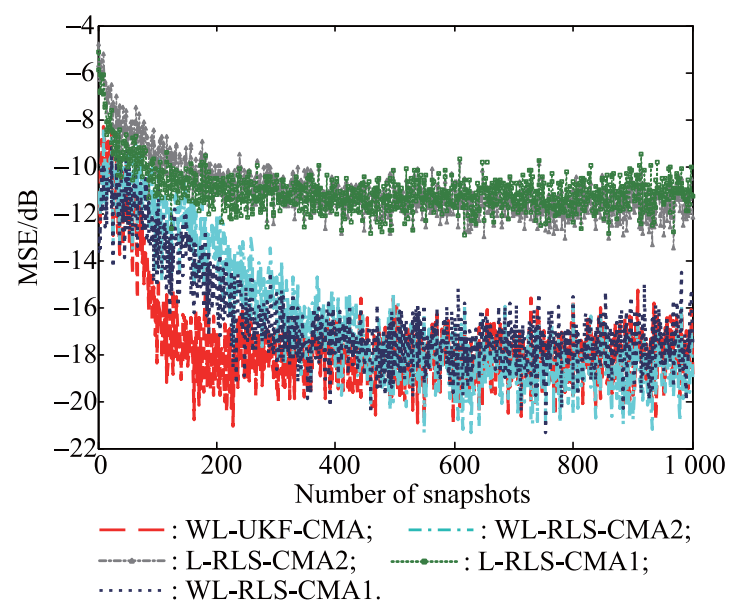

Fig. 9 Output MSEs of WL-UKF-CMA and LMS-type methods in Case 2

\subsection{WL-UKF-CMA vs RLS-type methods}

In this example, we examine the output SINRs and MSEs of WL-UKF-CMA, WL-RLS-CMA and L-RLS-CMA in
Case 1 and Case 2, respectively. Similar to the last example, for the WL solutions, we use "WL-RLS-CMA1" and "WL-RLS-CMA2" as the scenarios where the forgetting factor $\lambda$ is set to 0.98 and 0.998 , respectively. In a similar way, for the linear schemes, "L-RLS-CMA1" and "LRLS-CMA2" correspond to the scenarios of $\lambda=0.98$ and $\lambda=0.998$, respectively.

The output SINRs of WL-UKF-CMA, WL-RLSCMA1, WL-RLS-CMA2, L-RLS-CMA1 and L-RLSCMA2 in Case 1 are shown in Fig. 10. We observe in Fig. 10 that WL-UKF-CMA nearly approaches the same steady-state output SINR as WL-RLS-CMA2 and has a higher steady-state one than WL-RLS-CMA1, L-RLSCMA1 and L-RLS-CMA2. Moreover, we see that WLRLS-CMA1 has a faster convergence speed than WL-RLSCMA2, and L-RLS-CMA1 converges faster than L-RLSCMA2. That is due to the fact that a larger forgetting factor leads to a faster convergence speed and a lower steady-state output SINR.

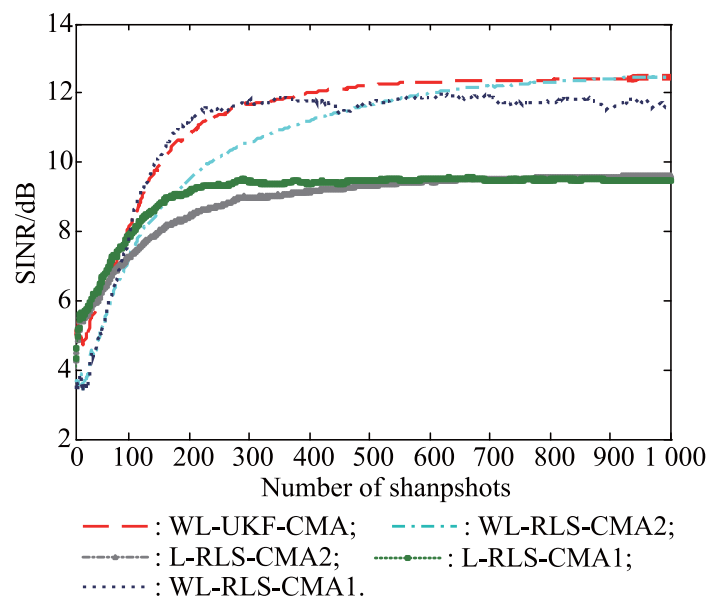

Fig. 10 Output SINRs of WL-UKF-CMA and RLS-type methods in Case 1

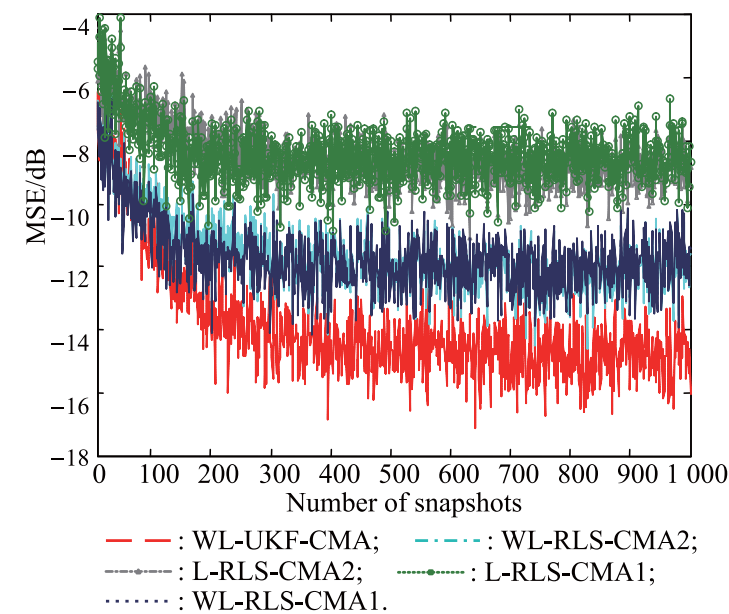

Fig. 11 Output MSEs of WL-UKF-CMA and RLS-type methods in Case 1 
In the contrary, a smaller forgetting factor results in a slower convergence speed and a higher output SINR. Fig. 11 shows the MSEs of WL-UKF-CMA, WL-RLSCMA1, WL-RLS-CMA2, L-RLS-CMA1 and L-RLSCMA2 in Case 1. From Fig. 11, we observe that WL-UKFCMA has the lowest steady-state output MSE among these counterparts, and the WL-scheme methods yield lower MSEs than their linear ones. It is noted that L-RLS-CMA1 has a higher steady-state output SINR than L-RLS-CMA2.

In Fig. 12, we show the output SINRs of WL-UKFCMA, WL-RLS-CMA1, WL-RLS-CMA2, L-RLS-CMA1 and L-RLS-CMA2 in Case 2. From Fig. 12, we see that, similar to Fig. 10, WL-UKF-CMA still has a faster convergence speed and a higher steady-state output SINR than its counterparts. Furthermore, it is seen that WL-RLS-CMA1 converges faster but reaches a lower steady-state SINR than WL-RLS-CMA2, and L-RLS-CMA1 has a faster convergence behavior but yields a lower SINR than L-RLSCMA2. That illuminates that the forgetting factor has an important influence on RLS-type methods.

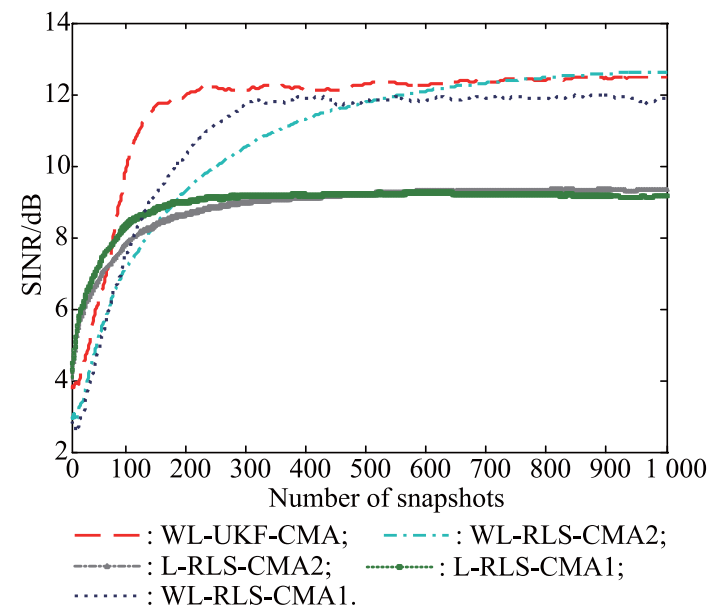

Fig. 12 Output SINRs of WL-UKF-CMA and RLS-type methods in Case 2

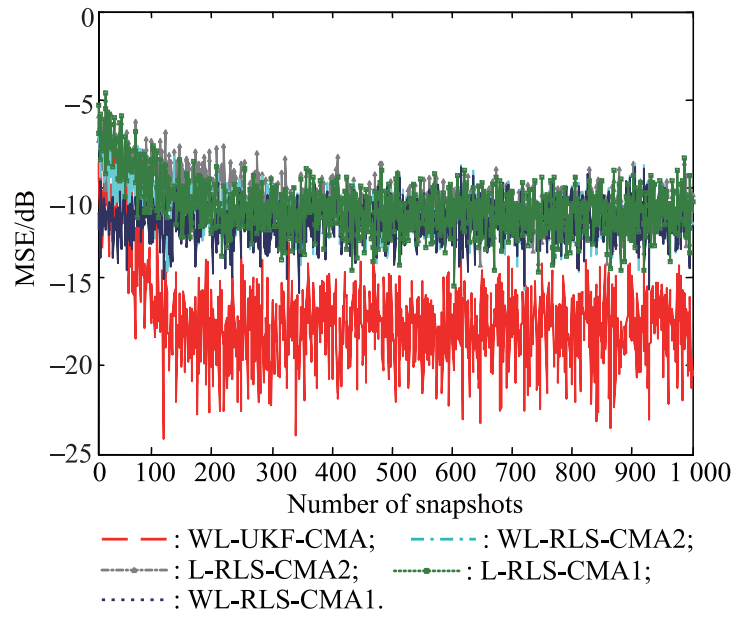

Fig. 13 Output MSEs of WL-UKF-CMA and RLS-type methods in Case 2
However, the proposed algorithm makes adjustment parameter be calculated adaptively by using the observed data and hence make it be used conveniently. The numerical curves of the output MSEs of WL-UKF-CMA and RLS-type methods in Case 2 are shown in Fig. 13. We observe in Fig. 13 that WL-UKF-CMA still yields the lowest MSE among the five methods compared. Therefore, the proposed algorithm has a faster convergence speed, a higher output SINR and a lower output MSE than its counterparts.

\section{Conclusions}

In this paper, WL-UKF-CMA has been proposed for WL blind adaptive beamforming. Besides using the constant modulus feature of the SOI, the proposed algorithm takes full advantage of the second-order noncircular property of the SOI, eventually resulting in considerable performance improvements. The simulation results show that WL-UKFCMA not only yields a faster convergence speed but also has a higher steady-state output SINR and a lower steadystate output MSE than L-UKF-CMA, LMS-type and RLStype methods. Unlike the MMSE-based or the linear constraint condition-based methods, the only a priori information of WL-UKF-CMA is the noncircular and constant modulus property of the SOI and hence makes it applied conveniently.

\section{References}

[1] P. J. Schreier, L. L. Scharf. Statistical signal processing of complex-valued data: the theory of improper and noncircular signals. Cambridge: Cambridge University Press, 2010.

[2] J. Zhang, L. Huang, L. Zhang, et al. Robust widely linear beamformer based on a projection constraint. Proc. of the IEEE International Conference on Acoustics, Speech and Signal Processing, 2015: 2509-2513.

[3] Y. Wang, L. Huang, Y. Shi. Robust widely linear reduced-rank adaptive beamforming based on joint iterative optimization. Proc. of the 4th International Conference Instrumentation and Measurement, Computer, Communication and Control, 2014: $366-369$.

[4] D. Xu, L. Huang, X. Xu, et al. Widely linear MVDR beamformers for noncircular signals based on time-averaged second-order noncircularity coefficient estimation. IEEE Trans. on Vehicular Technology, 2013, 62(7): 3219-3227.

[5] N. Song, J. Steinwandt, L. Wang, et al. Non-data-aided adaptive beamforming algorithm based on the widely linear auxiliary vector filter. Proc. of the IEEE International Conference on Acoustics, Speech and Signal Processing, 2011: 26362639.

[6] P. Chevalier, A. Blin. Widely linear MVDR beamformers for the reception of an unknown signal corrupted by noncircular interferences. IEEE Trans. on Signal Processing, 2007, 55(11): $5323-5336$.

[7] P. Chevalier, J. P. Delmas, A. Oukaci. Optimal widely linear MVDR beamforming for noncircular signals. Proc. of the IEEE International Conference on Acoustics, Speech and Signal Processing, 2009: 3573-3576. 
[8] R. Gooch, J. Lundell. The CM array: an adaptive beamformer for constant modulus signals. Proc. of the IEEE International Conference on Acoustics, Speech, and Signal Processing, 1986: $2523-2526$.

[9] P. J. Schreier, L. L. Scharf. Second-order analysis of improper complex random vectors and processes. IEEE Trans. on Signal Processing, 2003, 51(3): 714-725.

[10] N. Song, W. U. Alokozai, R. C. De Lamare, et al. Adaptive widely linear reduced-rank beamforming based on joint iterative optimization. IEEE Signal Processing Letters, 2014, 21(3): $265-269$.

[11] L. Qiu, Y. Cai, M. Zhao. Low-complexity variable forgetting factor mechanisms for adaptive linearly constrained minimum variance beamforming algorithms. IET Signal Processing, 2015, 9(2): 154-165.

[12] P. Chevalier, J. P. Delmas, A. Oukaci. Properties, performance and practical interest of the widely linear MMSE beamformer for nonrectilinear signals. Signal Processing, 2014, 97: 269 281.

[13] N. Song, R. C. De Lamare, M. Haardt, et al. Adaptive widely linear reduced-rank interference suppression based on the multistage wiener filter. IEEE Trans. on Signal Processing, 2012, 60(8): $4003-4016$.

[14] T. Zhang, B. Wang, S. Liu. Widely linear RLS constant modulus algorithm for complex-valued noncircular signals. Journal of Electronics (China), 2014, 31(5): 416-426.

[15] L. Wang, R. C. De Lamare. Constrained constant modulus RLS-based blind adaptive beamforming algorithm for smart antennas. Proc. of the IEEE 4th International Symposium on Wireless Communication Systems, 2007: 657-661.

[16] R. C. De Lamare, R. Sampaio-Neto, M. Haardt. Blind adaptive constrained constant-modulus reduced-rank interference suppression algorithms based on interpolation and switched decimation. IEEE Trans. on Signal Processing, 2011, 59(2): $681-695$.

[17] A. Hakkarainen, J. Werner, M. Valkama. RF imperfections in antenna arrays: response analysis and widely-linear digital beamforming. Proc. of the IEEE Radio and Wireless Symposium, 2013: $187-189$.

[18] H. Qian, K. Liu, W. Wang. Shrinkage widely linear recursive least square algorithms for beamforming. IEICE Trans. on Communications, 2016, 99(7): 1532-1540.

[19] S. C. Douglas. Widely-linear recursive least-squares algorithm for adaptive beamforming. Proc. of the IEEE International Conference on Acoustics, Speech and Signal Processing, 2009: $2041-2044$.

[20] X. Kang, J. Yang, M. Shuo. Blind adaptive receiver based on RLS-CMA for TH-UWB system. The Journal of China Universities of Posts and Telecommunications, 2011, 18(6): $38-$ 43.

[21] Y. Chen, T. Le-Ngoc, B. Champagne, et al. Recursive least squares constant modulus algorithm for blind adaptive array. IEEE Trans. on Signal Processing, 2004, 52(5): 1452 - 1456.

[22] L. Wang, R. C. De Lamare. Constrained adaptive filtering algorithms based on conjugate gradient techniques for beamforming. IET Signal Processing, 2010, 4(6): 686-697.

[23] J. Steinwandt, R. C. De Lamare, L. Wang, et al. Widely linear adaptive beamforming algorithm based on the conjugate gradient method. Proc. of the International ITG Workshop on Smart Antennas, 2011: 1-4.

[24] Y. H. Chen, C. T. Chiang. Adaptive beamforming using the constrained Kalman filter. IEEE Trans. on Antennas and Pro- pagation, 1993, 41(11): 1576-1580.

[25] A. El-Keyi, T. Kirubarajan, A. B. Gershman. Robust adaptive beamforming based on the Kalman filter. IEEE Trans. on Signal Processing, 2005, 53(8): $3032-3041$.

[26] J. S. Hu, M. T. Lee. Norm-constrained capon beamforming using multirank signal models with Kalman filter implementation. IEEE Trans. on Antennas and Propagation, 2014, 62(9): $4574-4583$.

[27] S. J. Julier, J. K. Uhlmann. Unscented filtering and nonlinear estimation. Proceedings of the IEEE, 2004, 92(3): 401-422.

[28] M. Z. A. Bhotto, I. V. Bajic. Constant modulus blind adaptive beamforming based on unscented Kalman filtering. IEEE Signal Processing Letters, 2015, 22(4): $474-478$.

[29] J. Yang, R. C. De Lamare. Widely-linear minimum-meansquared error multiple-candidate successive interference cancellation for multiple access interference and jamming suppression in direct-sequence code-division multiple-access systems. IET Signal Processing, 2015, 9(1): 73-81.

[30] Y. Cai, R. C. De Lamare, M. Zhao, et al. Low-complexity variable forgetting factor mechanism for blind adaptive constrained constant modulus algorithms. IEEE Trans. on Signal Processing, 2012, 60(8): $3988-4002$.

[31] Y. Cai, R. C. De Lamare. Low-complexity variable step-size mechanism for code-constrained constant modulus stochastic gradient algorithms applied to CDMA interference suppression. IEEE Trans. on Signal Processing, 2009, 57(1): $313-$ 323.

[32] E. A. Wan, R. Van Der Merwe. The unscented Kalman filter for nonlinear estimation. Proc. of the IEEE Adaptive Systems for Signal Processing, Communications, and Control Symposium, 2000: $153-158$.

[33] D. N. Godard. Self-recovering equalization and carrier tracking in two-dimensional data communication systems. IEEE Trans. on Communications, 1980, 28(11): 1867-1875.

[34] H. H. Zeng, L. Tong, C. R. Johnson. Relationships between the constant modulus and Wiener receivers. IEEE Trans. on Information Theory, 1998, 44(4): $1523-1538$.

[35] Y. L. Wang, Q. J. Ding, R. F. Li. Adaptive array processing. Beijing: Tsinghua University Press, 2009.

[36] M. L. Hong, W. Xiao. Performance of reduced-rank linear interference suppression. IEEE Trans. on Information Theory, 2001, 47(5): 1928 - 1946.

[37] R. C. De Lamare, R. Sampaio-Neto. Adaptive reduced-rank processing based on joint and iterative interpolation, decimation, and filtering. IEEE Trans. on Signal Processing, 2009, 57(7): $2503-2514$.

[38] R. C. De Lamare, R. Sampaio-Neto. Reduced-rank adaptive filtering based on joint iterative optimization of adaptive filters. IEEE Signal Processing Letters, 2007, 14(12): 980-983.

\section{Biographies}

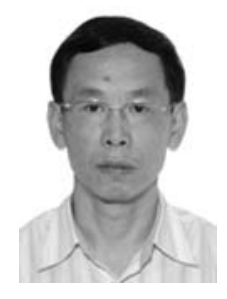

Huaming Qian was born in 1965 . He received his M.S. and Ph.D. degrees in 1990 and 2004, respectively, from Harbin Engineering University. He works in College of Automation, Harbin Engineering University, where he is currently a professor. His research interests include array signal processing, adaptive filter, wireless communications and networking, convex optimization, fault diagnosis and fault tolerant control. E-mail: qianhuam@sina.com 


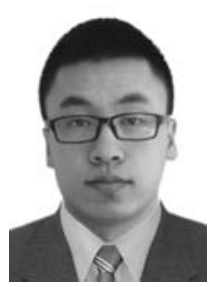

Ke Liu was born in 1986. He received his B.S. degree in 2010 from Harbin University of Science and Technology and his M.S. degree in 2013, from College of Automation, Harbin Engineering University. $\mathrm{He}$ is currently pursuing his Ph.D. degree in Harbin Engineering University. Since December 2016, he has been a joint training Ph.D. student, sponsored by the China Scholarship Council, in Temple University. His research interests include array signal processing, adaptive filter and convex optimization.

E-mail: tom.jim007@163.com

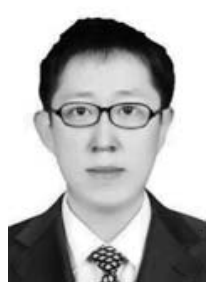

Long Li was born in 1984. He received his M.S. degree in 2013, from College of Automation, Harbin Engineering University. He is currently an engineer in Information Communication Branch of State Grid Heilongjiang Electric Power Company Limited. His research interests include wireless communications and networking, ship motion control and information fusion.

E-mail: 56630170@qq.com

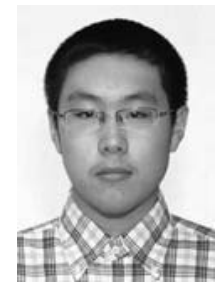

Linchen Qian was born in 1995. He is currently pursuing his B.S. degree in School of Computing, Beihang University. His research interests include artificial intelligence and signal processing.

E-mail: qianlc95@sina.com

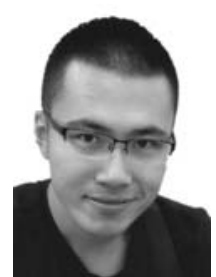

Junda Ma was born in 1988. He received his B.S. degree in 2010 from Harbin University of Science and Technology and his M.S. degree in 2013, from College of Automation, Harbin Engineering University where he is currently pursuing his Ph.D. degree. His research interests include cooperative control of autonomous surface vehicles, nonlinear control system and signal processing.

E-mail: 982564224@qq.com 